\title{
EVALUACIÓN DEL ESTADO DE LA MADERA EN OBRAS DE REHABILITACIÓN MEDIANTE TÉCNICAS DE ULTRASONIDOS Y OBTENCIÓN DE PARÁMETROS RESISTENTES
}

\author{
(EVALUATION OF WOOD CONSERVATION WORKS BY ULTRASOUND TECHNIQUES AND \\ OBTENTION OF RESISTANCE PARAMETERS)
}

Carmen Rodriguez Liñán, Dra. Arquitecta. Catedrática de Construcción

Paloma Rubio de Hita, Arquitecta. Becaria F.P.I.

Dpto. de Construcciones Arquitectónicas I- E. T. S. A. (Sevilla)

ESPAÑA

\section{RESUMEN}

Este articulo presenta el desarrollo de un método de ensayo, no destructivo, para la inspección y evaluación in situ de elementos estructurales de madera, basado en las medidas de la velocidad de ondas ultrasónicas y determinación de humedad. Mediante la aplicación de este método se podrá obtener una estimación de la resistencia a flexión y del módulo de deformación de la madera estudiada, asi como la detección de estados de deterioro de la misma. Esto aportará información suficiente a la hora de tomar decisiones en una obra de rehabilitación con elementos estructurales de este material.

\section{$S(M M M A R Y$}

A nondestructive technique for inspection and evaluation inplace timber structures and components based on ultrasonic pulse velocity, and moisture content is presented herein. By applying this methodology we could obtain the bending strength and elasticity modulus of timber beams, as well as the damage in them. The cuantitative determination of the existing strength of timber beams will provide information necessary to take the most suitable decisions in rehabilitation works involving timber structural elements.

\section{DEFINICIÓN DEL PROBLEMA Y OBJETIVOS PROPUESTOS}

Se puede suponer y admitir que la causa más importante de decisiones equivocadas en cualquier proceso, dando por supuesta la capacitación del o de los individuos que toman las decisiones, es una falta de información o de métodos adecuados que le lleven a una correcta interpretación de la realidad, o de la toma de datos que constituyen la base sobre la cual se van producir las decisiones.
Es en el campo de la realización de un proyecto y de la dirección de obras donde se presenta, con gran frecuencia, la necesidad de tomar decisiones sobre la bondad (resistencia) de un material desde el punto de vista del uso y durabilidad. Si esto es cierto para cualquier tipo de proyectos, lo es aún más si se trata de una obra o proyecto de rehabilitación, reparación o restauración.

En el campo concreto que hemos investigado nos planteamos la cuestión de estimar la resistencia de la madera en una 
obra de rehabilitación sin necesidad de recurrir a ensayos destructivos, utilizando una técnica de fácil manejo como es la de los ultrasonidos.

El problema a resolver no es sólo éste, se trata de obtener también una medida del grado de pudrición de la madera, desarrollando una metodología de ensayo utilizando la técnica de ultrasonidos que nos ayude en la toma de decisiones acerca de la necesidad de reparar o sustituir un determinado elemento constructivo de madera. La problemática de la pudrición de la madera debidaa agentes bióticos (hongos de pudrición) es una hecho muy extendido en las obras de rehabilitación, donde aparece la madera como elemento estructural. Esta patología se presenta asociada a un elevado grado de humedad de la madera y a una localización en las cabezas empotradas de las vigas y en las zonas adyacentes. La cuestión está en responder, mediante un ensayo no destructivo: ¿Hasta dónde se extiende la zona deteriorada afectada por la pudrición? ¿Es necesario descubrir el apoyo empotrado para comprobar el estado del empotramiento?

Actualmente, el empleo de ondas ultrasónicas para la realización de ensayos no destructivos está muy extendido en el estudio de materiales (metales, hormigón, materiales compuestos, etc. ) en los que estos ensayos proporcionan resultados muy fiables. Este hecho, y el conjunto de investigaciones previas existentes que hemos analizado en el estado de la cuestión, nos han llevado a plantearnos, como método de ensayo no destructivo para el estudio de la madera en las obras de rehabilitación, la medida de la transmisión ultrasónica a través del material

Por lo tanto, los objetivos que nos hemos propuesto en el presente trabajo de investigación es dar respuesta a la problemática anteriormente descrita y son:

A) Desarrollar un método de ensayo no destructivo para la inspección y evaluación, in situ, del estado de elementos estructurales de madera, basándonos en las medidas de la velocidad de transmisión de ondas ultrasónicas.

B) Obtener una estimación del valor de la resistencia a flexión y del módulo de deformación estático de la madera estudiada a partir de las mediciones de ultrasonidos, utilizando las correlaciones obtenidas en laboratorio entre medidas de velocidad de transmisión y valores de tensión de rotura aflexión mediante ensayos normalizados.

Además de estos objetivos generales o primarios, existen otros objetivos intermedios, necesarios para alcanzar los dos anteriores, pero no por eso de menor validez o interés científico:

Estudiar el comportamiento de la madera, tanto vieja como nueva, frente a la transmisión de ondas ultrasónicas, para lo cual hay que obtener: a) Influencia en la velocidad de transmisión de la posición de las fibras de la madera (velocidades normales, tangenciales y radiales).

b) Relación entre las velocidades de transmisión según las direcciones principales de la madera.

c) La influencia en las velocidades de transmisión de la humedad, temperatura y posibles defectos de la madera.

e) Una metodología de ensayos, mediante medidas de ultrasonidos, que permita conocer la posición de los anillos de crecimiento, para cuando no se tiene acceso a la sección del elemento.

f) Un banco de datos con los resultados de :

1.- Medidas de las velocidades de transmisión de ondas ultrasónicas, obtenidas para probetas de madera nueva en laboratorio.

2.- Valores de las tensiones de rotura a flexión y módulos de deformación estática, obtenidos mediante ensayos de rotura sobre las probetas anteriores.

Con el objeto de poder establecer las correlaciones existentes entre medidas de velocidades de transmisión y parámetros resistentes

\section{Grado de innovación e interés de los objetivos propuestos en relación a otros métodos conocidos}

El desarrollo de los objetivos propuestos supone un gran avance en la inspección no destructiva de la madera. Hasta ahora, los métodos más extendidos para la evaluación de la madera y el establecimiento de calidades de la misma han sido:

\section{- La inspección visual.}

- La respuesta de la madera al ser pinchada por un punzón.

- El sonido emitido al ser golpeada por un martillo.

- Comportamiento al ser perforada por un taladro.

Los métodos de pinchar y taladrar son inspecciones destructivas y, por otro lado, no muy exactas, ya que hay que pinchar muchos puntos para obtener resultados fiables. Estos métodos, por otra parte, requieren bastante experiencia del trabajador operario y están sujetos a la subjetividad de su criterio. La normativa española, que basa la clasificación de las calidades de la madera en la inspección visual, considerándolas de mayor calidad en ausencia de defectos visibles, parece estar incompleta en el estudio de la madera, ya que muchos de los defectos y patologías de la misma no pueden ser apreciados des̄e el exterior, con lo que pueden falsearse los valores de resistencia que se asignan a una madera de una calidad determinada. 
El método que desarrollamos presenta la ventaja de dar unas medidas objetivas (velocidades de transmisión) que correlacionamos con parámetros resistentes o con estados de deterioro o pudrición.

Por otro lado, el método de ultrasonidos, sobre otros métodos no destructivos más sofisticados y poco utilizados como los rayos $\mathrm{X}$, tiene la ventaja de poder ser aplicable in situ, ya que el instrumental necesario para los ensayos es manejable, económico y de poco tamaño, cosa que no ocurre con los aparatos de rayos $\mathrm{X}$.

\section{Delimitación del ámbito y limites del trabajo}

Al exponer el ámbito hasta donde se ha actuado en este .trabajo queremos dejar constancia de que se encuentra inmerso en una investigación que podemos considerar no concluida, ya que los resultados de dicho trabajo de investigación serán ampliados en etapas posteriores.

Los límites de actuación se establecen en tres campos:

\section{A.1 Materiales: Tipos de madera}

Dentro de las especies de madera utilizadas en construcción (coniferas, frondosas), nuestro estudio se ha centrado en las maderas de coníferas, y más concretamente en la especie botánica conocida como Pinus Sylvestris. Esta especie es la que se utilizó con mayor frecuencia en los forjados de viviendas y corrales de vecinos de nuestra ciudad (Sevilla) que son objeto de trabajos de rehabilitación o restauración.

\section{A. 2 Elementos estructurales de madera estudiados}

Vigas y viguetas de forjados de madera

En etapas posteriores se ampliará el estudio a pilares y armaduras de cubiertas

\section{A.3 Estado de los materiales . Patología según el tipo de agente destructor}

Madera nueva. Sin deterioro ni patología.

Madera vieja. Procedente de una o varias obras de rehabilitación.

Deteriorada por alguno de los agentes siguientes:

- Agentes abióticos: Humedad, cambios bruscos de temperatura, acción del fuego.

- Agentes Bióticos:Insectos xilófagos, hongosxilófagos, bacterias.

\section{HIPÓTESIS DE PARTIDA}

Teniendo como referencia toda la bibliografia consultada y el estado de la cuestión, es posible desarrollar un método de ensayo no destructivo mediante la técnica de ultrasonidos que permita detectar estados de deterioro y pudrición en elementos de madera vieja, empotrados o no accesibles, para establecer criterios de sustitución o refuerzo en obras de rehabilitación.

Las medidas de velocidades de transmisión de ultrasonidos en maderas permiten obtener una medida cualitativa del grado de calidad de la madera, como ocurre con el mismo ensayo en el hormigón. Valores cuantitativos de resistencia sólo es posible obtenerlos mediante correlaciones estadísticas entre ensayos destructivos y no destructivos, obtenidas a partir de un banco de datos realizado en laboratorio con madera del mismo tipo de la que se estudia.

\section{ESTADO DE LA CUESTIÓN}

Dentro de los ensayos no destructivos utilizados en elementos y estructuras de madera, los instrumentos basados en la medición del impulso ultrasónico han sido utilizados con distintos grados de éxito en la evaluación del estado de la madera en estructuras en servicio desde 1966 (1).

Pero más recientemente, los estudios en el campo de la técnica de ultrasonidos, aplicados a la madera para detectar estados de deterioroy pérdidas de resistencia, han sido realizados por investigadores de importantes compañías japonesas, americanas y canadienses; las primeras como propietarias de grandes longitudes de líneas telefónicas realizadas con postes de madera, y las segundas por la gran cantidad de puentes realizados mediante pilotes de madera, necesitados de frecuentes y costosas labores de mantenimiento para detectar cualquier deterioro o pérdida de resistencia, que sería fatal para la seguridad de su utilización.

Hemos revisado toda la bibliografía existente y relacionada con el tema mediante un seguimiento a través de las bases de datos existentes.

Como resumen, podemos afirmar que un trabajo igual al que planteamos no está realizado, aunque existen estudios que nos pueden servir de referencia a modo de metodología a aplicar. Las investigaciones realizadas se refieren a elementos trabajando a compresión: pilotes de puentes de madera (2), donde se establecen correlaciones entre medidas de velocidades de ultrasonidos y resistencia a la compresión de pilotes de madera de pino amarillo. Arita y

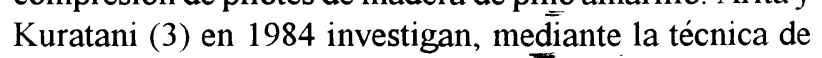
ultrasonidos, la resistencia residual de la base de un poste de madera de tendido eléctrico con madera de cedro 
japonés, en la cual se conoce que la zona de pudrición se localiza en la albura del árbol. Wayne y Wilcox (4) aportan en sus investigaciones relaciones entre la velocidades de transmisión y pérdida de peso de madera de pino, sometida en laboratorio a la acción de hongos causantes de la pudrición cúbica para distintas frecuencias de transmisión.

En Europa se pueden destacar los trabajos de J.L. Sandoz (5), investigador de L' École Polytéchnique Federal de Lausanne que, en 1990, lee su tesis sobre "La validez del ensayo de ultrasonido aplicado a la selección y clasificación de maderas de construcción de pinos y abetos centroeuropeos".

En España hay que destacar los trabajos de Arriaga y Argüelles (A.I.T.I.M) (6) relativos a normativas de cálculo de madera aserrada y encolada y en sus investigaciones de evaluación de forjados antiguos de madera mediante la técnica de ultrasonidos

También destacamos los trabajos realizados por Palaia y Galvañ (7) sobre la aplicación de los ultrasonidos a la determinación de los estados de deterioro. Sus investigaciones están basadas en el análisis de las señales de un osciloscopio incorporado al aparato de ultrasonidos, y se realizan sobre maderas con pudrición simulada mediante perforaciones realizadas en laboratorio.

\section{ESQUEMA DEL PLAN EXPERIMENTAL REALIZADO}

\subsection{Equipos Utilizados}

- Ensayos de contenido de humedad en laboratorio, según norma UNE 5652977 mediante desecación hasta el estado anhidro

Estufa con temperatura de secado de $110^{\circ}$ y balanza de precisión de pesada de $0,001 \mathrm{gr}$

- Determinación de humedad in situ:

Higrómetro de resistencia modelo Timbermaster D378T, de la marca Protimeter ("sonda")

Higrómetro de resistencia modelo M49, de la marca P\&S Laboratories ("M49").

- Ensayos de ultrasonidos:

Aparato de ultrasonidos modelo BPV, de la marca Krautkrämrer. Medición entre 0,1 y 9999,9 $\mu \mathrm{s}$, precisión de $0,1 \mu \mathrm{s}$

Palpadores cónicos de cristales piezoeléctricos de circonato de plomo titanio, con frecuencia de emisión de $50 \mathrm{kHz}$.
- Ensayos de rotura a flexión, según norma UNE 56-537 79

Máquina multiensayo servocontrolada modelo MEM101/M4, de la casa Suzpecar, S.L.

\subsection{Ensayos no destructivos en madera nueva. Fase I}

\section{BANCO DE DATOS}

A) Toma de datos y defectos:

Sobre probetas de madera nueva de pinus silvestre, de dimensiones $10 \times 15 \times 30 \mathrm{~cm}$ (probetas Tipo b).

B) Medidas de ultrasonidos:

Sobre las probetas (Tipo b) de toma de datos y defectos

Sobre las probetas (Tipo a) utilizadas para los ensayos de flexión $(2 \times 2 \times 30 \mathrm{~cm})$ y para distintos valores de humedad

C) Determinaciones de humedad:

En las probetas (Tipo b) se realizan mediante sonda "Protimeter" y con el M49. Las medidas se llevan a cabo en ambos extremos de la probeta y por todas sus caras, obteniendo una media en cada uno de los extremos y una media para la probeta.

En las probetas (Tipo a) la determinación de humedad se ha hecho por desecación en estufa, después de llevarlas hasta el grado de saturación, según norma UNE 56529 77 para llegar al estado anhidro

D ) Determinaciones de densidad:

Para las probetas ( Tipo a), después de realizar los ensayos de flexión, haciéndose una determinación de humedad y densidad, según norma UNE 56-529 77 y UNE 56-531 77 ( en pequeñas probetas de $2 \times 2 \times 3 \mathrm{~cm}$ )

4.3 Ensayos no destructivos realizados en obra de rehabilitación. Madera vieja. Fase II

\section{BANCO DE DATOS EN MADERA VIEJA}

Tipos de ensayos: Medidas de ultrasonidos y determinaciones de humedad con sonda Protimeter y M49.

Muestra:Vigas y viguetas de madera en obras de rehabilitación

A ) Ensayo 1. Homogeneidad de la sección:

En este ensayo, y para cada sección, se realizan tres medidas de ultrasonidos ( $\mathrm{T} 1, \mathrm{~T} 2, \mathrm{~T} 3)$, enfrentando los 
palpadores como se indica en la figura 1 , al mismo tiempo que se efectúan determinaciones de humedad con los dos aparatos en los mismos puntos.

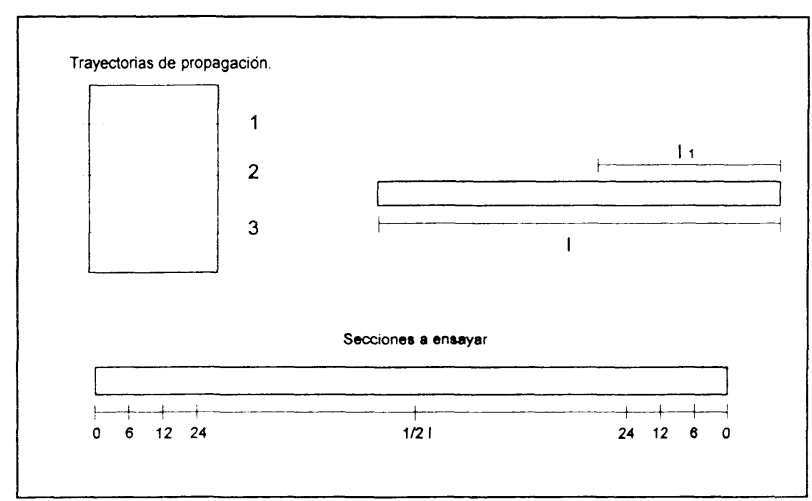

Fig. 1.-Ensayo 1 de homogeneidad dela sección. Posición de palpadores.

B) Ensayo 2. Determinación de la posición de la fibra:

En algunos casos puede ser necesario conocer la disposición de los anillos de crecimiento; esto se consigue realizando 5 medidas de velocidades de ultrasonidos, tal y como puede apreciarse en la figura 2; la mayor de ellas nos determina la posición de los anillos de crecimiento.

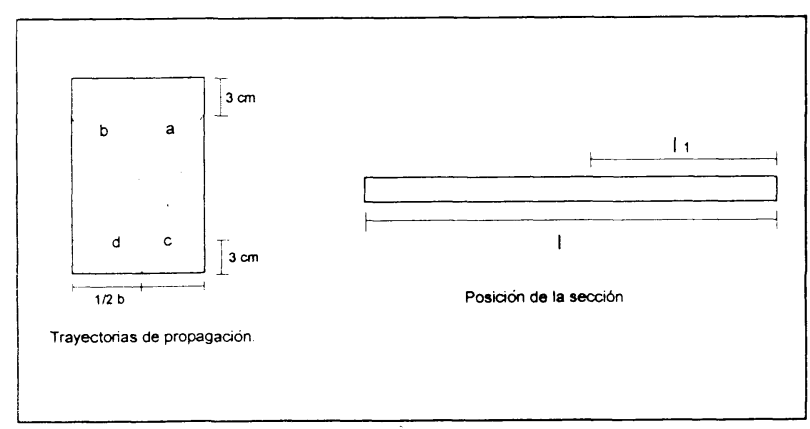

Fig. 2,- Ensayo 2. Determinación de la posición de la fibra.

C) Ensayo 3. Mediciones longitudinales y cruzadas:

Las mediciones con ultrasonidos en el sentido longitudinal de la fibra no se pueden realizar en las vigas de forjados, ya que las cabezas de éstas se encuentran empotradas. Para sustituir estas medidas se realizan otras cruzadas, situando los palpadores en caras enfrentadas en el tercio central de la viga (Fig. 3).

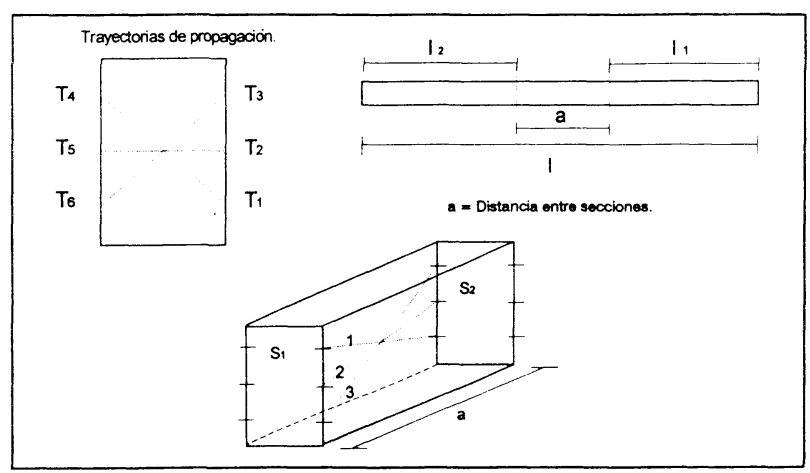

Fig. 3.- Ensayo 3. Medidas longitudinales cruzadas.
D) Organización del plan de ensayos:

A la hora de organizar el plan de ensayos en una obra de rehabilitación determinada lo haremos en función del estado de la estructura a estudiar.

\section{MEDIDAS EN VIGAS Y VIGUETAS DE MADERA}

1. Estructura en buen estado aparente de conservación

Plan de ensayos dirigido a confirmar que el buen estado de conservación coincide con el real.

- Medidas longitudinales:

Medidas de ultrasonidos en el tercio central, mediante medidas cruzadas, ensayo $n^{\circ} 3$. (Velocidades de transmisión y humedad) Tamaño de la muestra: $N^{o}$ mínimo vigas medidas $N \geq 6$

- Medidas transversales: ensayo 1 de homogeneidad de la sección:

Medidas en la sección central del vano.

Medición en los extremos de las vigas próximos a los apoyos

\section{Estructura con patología debida a agentes bióticos}

En este caso, lo que interesa es delimitar su extensión para localizar zonas de actuación.

Se harán medidas transversales, mediante un barrido por secciones contiguas cada $10 \mathrm{~cm}$, a la zona afectada (Ensayo 1).

Si el ataque está visiblemente generalizado por todo el elemento, se tomarán algunas medidas, sólo como dato a archivar, para conocer la reducción de velocidad asociada $a$ un determinado agente

\section{Patología debida a agentes abióticos}

La acción del fuego o cambios bruscos de humedad provocan fendas y fisuras. En el caso de la existencia de fendas y fisuras, lo que nos interesa conocer es la resistencia de estos elementos. Para ello se representaron los defectos y su situación .

Si la estructura está atacada por el fuego es necesario determinar la sección residual resistente para determinar su sustitución o no. Ensayos 1 y 3 .

\subsection{Ensayos destructivos. Fase III}

Los ensayos destructivos realizados tan consistido en la rotura a flexión estática de probetas normalizadas según la 
norma UNE 56537-77 en la maquina de ensayo MEN 101/ M4 de la casa Suzpecar, S. L.

Los ensayos se han hecho, tanto para madera vieja como para nueva, ambas de la madera seleccionada de pino flandes.

Madera nueva: De cada probeta (Tipo b) utilizada para los ensayos de ultrasonidos se han obtenido un mínimo de 5 probetas (Tipo a) para el ensayo a flexión; $n^{\circ}$ de muestras obtenidas: $55 ; \mathrm{n}^{\circ}$ de muestras ensayadas: 37 .

Madera vieja: De la obra de rehabilitación estudiada a lo largo del presente trabajo se han obtenido muestras de madera de vigas que se habían desmontado para su sustitución. De las partes sanas de las mismas se han tallado probetas siguiendo la norma UNE, análogamente a como se ha hecho en madera nueva; $\mathrm{n}^{\circ}$ de muestras obtenidas: $6, \mathrm{n}^{\circ}$ de muestras ensayadas: 6 .

\section{RESULTADOS DE LOS ENSAYOS. FASE IV}

\subsection{Madera nueva}

1. Relación de velocidades según las distintas direcciones de transmisión:
Se han estudiado las relaciones que existen entre las velocidades de transmisión, según las direcciones principales y según distintos valores de humedad de las probetas (Tablas 1 y 2).

$\mathrm{V}_{\mathrm{L}}$ : Velocidad en la dirección de las fibras.

$\mathrm{V}_{\mathrm{N}}$ : Velocidad en dirección radial o normal a los anillos de crecimiento.

$\mathrm{V}_{\mathrm{T}}$ : Velocidad en dirección tangente a los anillos de crecimiento.

$\mathrm{V}_{\text {CRUz }}$ : Velocidad cruzada entre caras opuestas no adyacentes.

Para los dos tipos de probetas: (Tipo a) y (Tipo b), se han estudiado las relaciones y sus parámetros estadísticos:

$1^{\circ}$ ) Para cada tipo de probeta.

$2^{\circ}$ ) Para el conjunto de valores obtenidos para los dos tipos

2. Método de determinación de la posición de los anillos de crecimiento mediante medidas transversales:

Tabla 1

Resultados globales (parámetros estadísticos), probetas $(a+b)$

\begin{tabular}{|c|c|c|c||}
\hline Número de valores $N$ & $\begin{array}{c}\text { Valor medio } \\
\mathrm{V}_{\mathrm{L}} / \mathrm{V}_{\mathrm{N}}\end{array}$ & Desviación típica $\sigma_{\mathrm{n}-1}$ & $\begin{array}{c}\text { Error normal } \\
u=\sigma_{\mathrm{n}-1} / \text { media }\end{array}$ \\
\hline 28 & 2,459 & 0,20 & 0,081 \\
\hline \hline Número de valores $\mathrm{N}$ & $\begin{array}{c}\text { Valor medio } \\
\mathrm{V}_{\mathrm{L}} / \mathrm{V}_{\mathrm{N}}\end{array}$ & Desviación típica $\sigma_{\mathrm{n}-1}$ & $\begin{array}{c}\text { Error normal } \\
=\sigma_{\mathrm{n}-1} / \text { media }\end{array}$ \\
\hline 28 & 3,44 & 0,20 & 0,058 \\
\hline Número de valores $\mathrm{N}$ & $\begin{array}{c}\text { Valor medio } \\
\mathrm{V}_{\mathrm{L}} / \mathrm{V}_{\mathrm{N}}\end{array}$ & Desviacion típica $\sigma_{\mathrm{n}-1}$ & $\begin{array}{c}\text { Error normal } \\
\sigma_{\mathrm{n}-1} / \text { media }\end{array}$ \\
\hline 28 & 1,4 & 0,074 & 0,052 \\
\hline
\end{tabular}

Tabla 2

Resultados probetas tipo b): Relación entre velocidad longitudinal y velocidad cruzadas máxima

\begin{tabular}{|c|c|c|c|}
\hline Número de valores $N$ & $\begin{array}{c}\text { Valor medio } \\
V_{L} / V_{N}\end{array}$ & Desviación típica $\sigma_{n-1}$ & $\begin{array}{c}\text { Error normal } \\
u=\sigma_{n-1} \Gamma \text { media }\end{array}$ \\
\hline 10 & 1,59 & 0,2 & 0,12 \\
\hline
\end{tabular}


A partir de los resultados obtenidos en el punto anterior es posible determinar la posición de los anillos de crecimiento cuando no hay posibilidad de acceder a las cabezas empotradas de las vigas (Fig. 4).

3. Influencia de la humedad en las velocidades de transmisión:

Se ha determinado el porcentaje de variación de las velocidades según las distintas direcciones $\left(\mathrm{V}_{\mathrm{L}}, \mathrm{V}_{\mathrm{N}}\right)$ cuando la humedad varía entre el $12 \%$ (humedad de referencia) y el $28 \%$ ( humedad de saturación ).

Se ha establecido la correlación que existe entre la velocidad longitudinal con la humedad y la velocidad normal con la humedad, para cuantificar qué corrección debe efectuarse a una velocidad cualquiera para que corresponda a las medidas standard, siempre referidas al $12 \%$ de humedad (Figs. 5 y 6).

\subsection{Ensayos no destructivos en madera vieja. Actuación en una obra de rehabilitación. Fase II}

La edificación es un antiguo corral de vecinos, situada en el casco histórico de Sevilla, en la c/ Narciso Campillo $\mathrm{n}^{\circ} 15$, en la antigua collación de la Magdalena. Edificio de

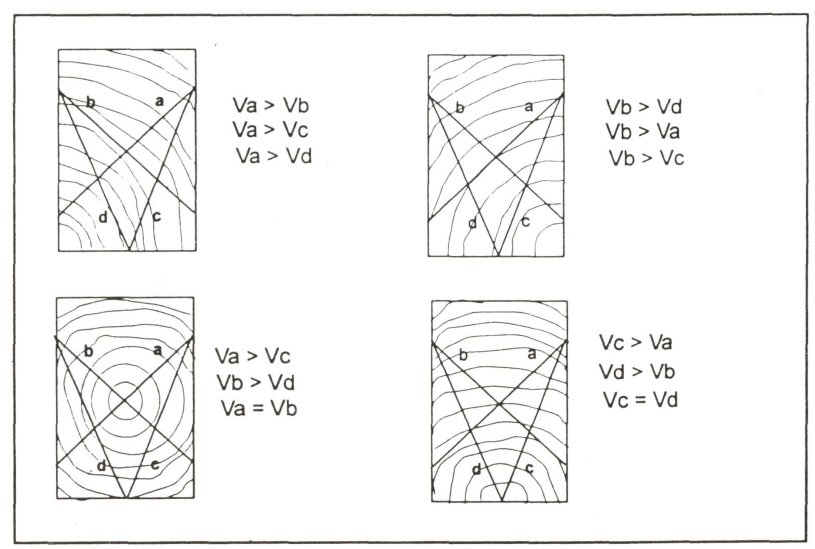

Fig. 4.-Determinación de la posición de los anillos de crecimiento según valores de velocidad de transmisión.

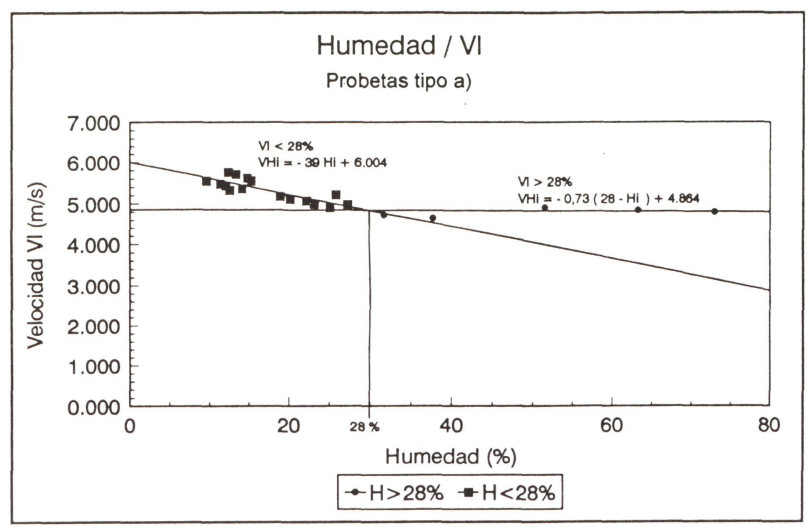

Fig. 5.- Relación entre $V_{L}$ y contenido de humedad para la totalidad de probetas (Tipo a).

(c) Consejo Superior de Investigaciones Científicas Licencia Creative Commons 3.0 España (by-nc)

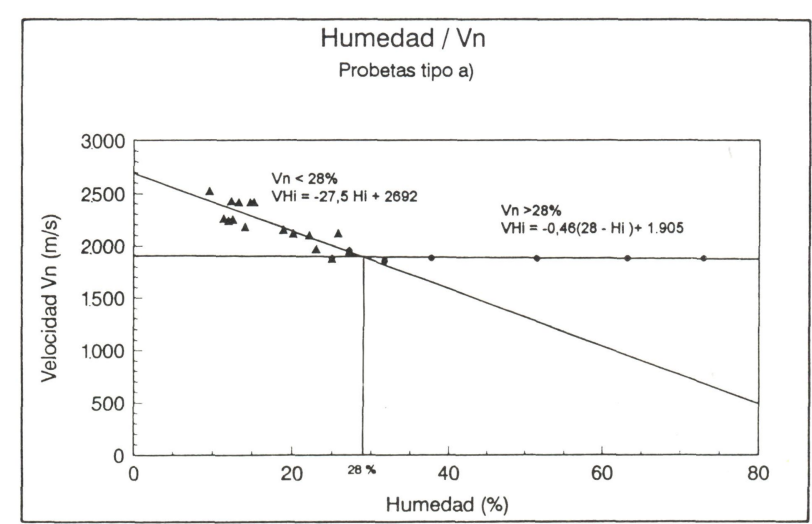

Fig. 6.- Relación entre $V_{N}$ y contenido de humedad para la totalidad de probetas (Tipo a).

dos plantas, con estructura de forjados de madera de pino flandes y muros de carga de fábrica de ladrillo (Fot.1).

Los forjados de madera del edificio(Fot. 2) están formados por viguetas de madera de pino flandes empotrados en

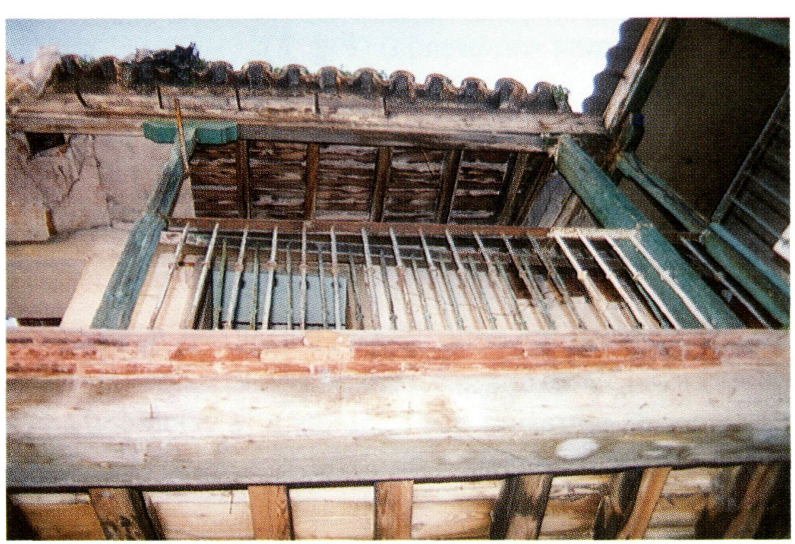

Foto 1.- Patio de la vivienda.

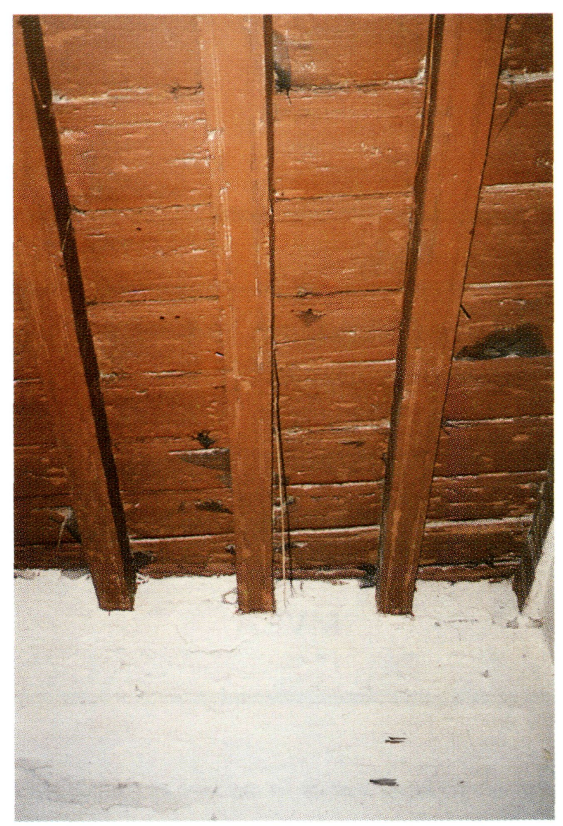

Foto 2.- Forjado de la habitación 1. 
muros de fábrica de ladrillo y el entrevigado está formado por tablazón de madera que se fija a las viguetas mediante grandes clavos "tipo gitanos". En sentido ortogonal a las viguetas existen unos listones a modo de zoquetes que se fijan mediante clavado y entalladura a las viguetas y sirven para sellar las uniones de la tablazón. Sobre la tablazón se dispone un relleno y la solería con su correspondiente mortero de agarre.

Después de una toma de datos previa, donde se recogían características y patologías detectadas, se elaboró un plan de ensayos no destructivos para la planta baja del edificio; con los criterios establecidos en el apartado anterior se llevó a cabo un estudio, según elementos y localización (Fig. 7 y Tabla 3).

$\left.1^{\circ}\right)$ Medidas de velocidades transversales en cada una de la secciones analizadas en las tres trayectorias descritas en el apartado de definición de ensayos.

$2^{\circ}$ ) Posición de la dirección de la fibra en cada viga, estimada a partir de los valores máximos de la velocidad transversal (zona sombreada) (Fig. 8).

$\left.3^{\circ}\right)$ Representación de los valores de humedad con los dos aparatos medidores (sonda y M49) en los puntos de la viga donde se han realizado las medidas de velocidades transversales.

$\left.4^{\circ}\right)$ Representación en la viga de los valores de velocidad referidos al $12 \%$ de humedad con ambos aparatos, utilizando las rectas de regresión que relacionan la velocidad con el porcentaje de humedad

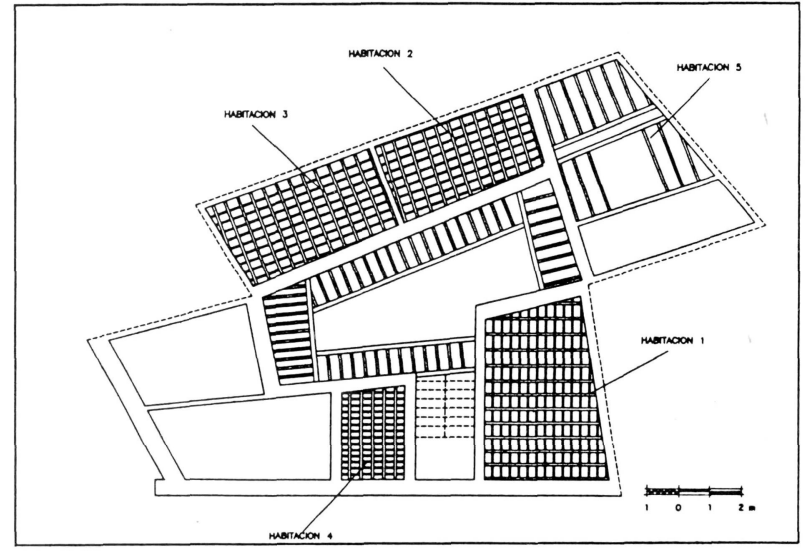

Fig. 7.- Esquema de plantas de forjados.

$5^{\circ}$ ) Valores de velocidad media en cada sección, referidos tanto a la humedad medida con sonda como con el M49 ${ }^{1}$

$\left.6^{\circ}\right)$ Representación gráfica, mediante diagrama de barras verticales, de los valores de la velocidad media en cada sección (Figs. 9 y 10).

En estas gráficas también se marca una recta correspondiente a una pérdida del $10 \%$ de la velocidad media transversal en el tramo central (velocidad máxima), ya que, según los estudios de Wilcox [1], una bajada del $10 \%$ de esta velocidad indica una pérdida de peso del $15 \%$ para ataque producido por los hongos causantes de la pudrición cúbica (G. Trabeum) y un $10 \%$ para hongos (Poria Placenta $)^{2}$.

Tabla 3

Localización de elementos

\begin{tabular}{||l|l||}
\hline Localización: & Elementos: \\
\hline Forjado Habitación H-1 & Viguetas 1-9 y 1-10 (desmontadas) \\
Forjado Habitación H-2 & Viguetas 2-2, 2-4 y 2-9 \\
Forjado Habitación H-3 & Vigueta 3-2 \\
Forjado Habitación H-4 & Vigueta 4-4 \\
Forjado Garaje & Vigueta (desmontada ) \\
Forjado Galería & Viga de borde (desmontada ) \\
$"$ " $\quad$ MVCA & Vigueta (desmontada ) \\
$" \quad$ " & Vigueta cepillada (desmontada ) \\
\hline
\end{tabular}

(1) Evidentemente la humedad de la madera es única; sin embargo, como se utilizan dos higrómetros con diferente tecnologia se obtienen medidas diferentes de humedad.

(2) Éste es el tipo de pudrición detectado en las viguetas de la obra. 


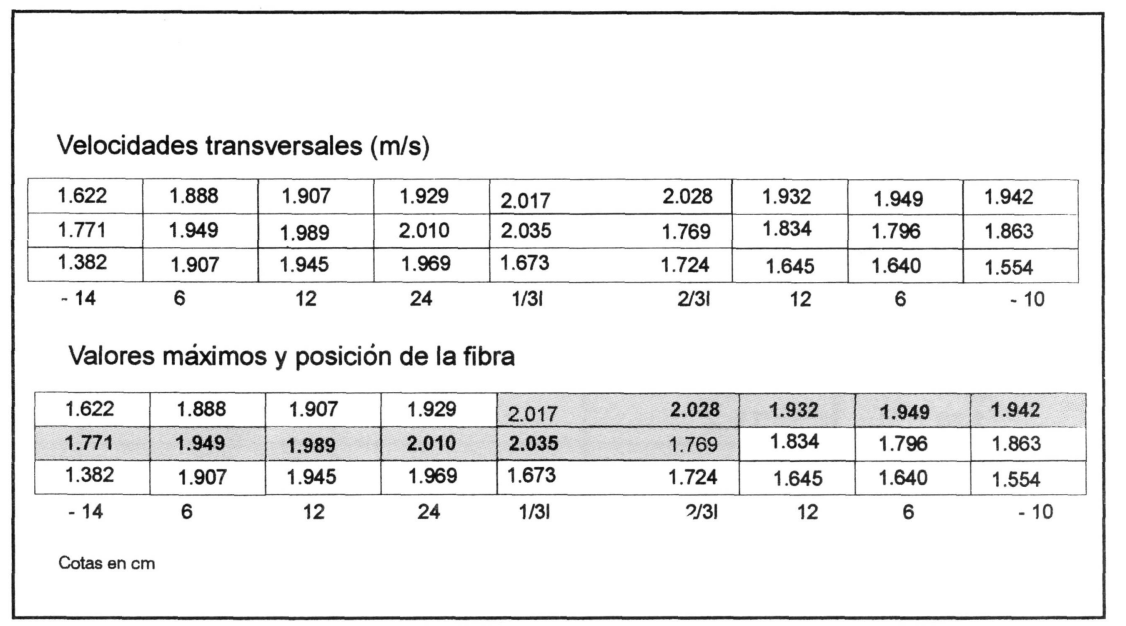

Fig. 8.- Medidas de velocidades transversales y determinación de la posición de la fibra en la vigueta $1-9$

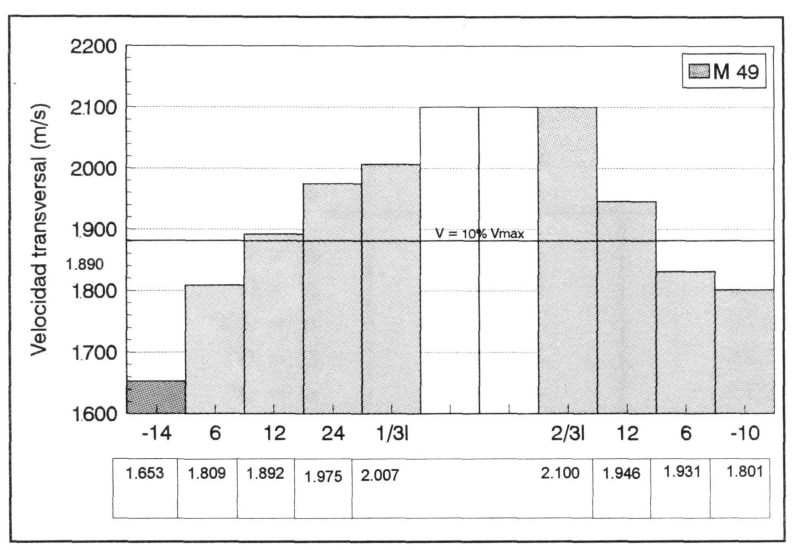

Fig. 9.- Velocidad transversal media correspondiente a cada sección referidas al $12 \%$ de humedad (valores de humedad obtenidos con el M49). (Vigueta 1-9).

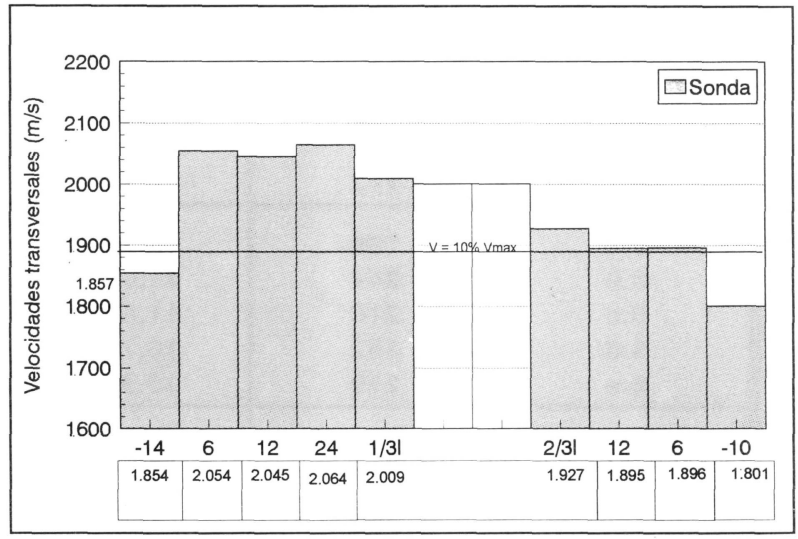

Fig. 10.- Velocidades transversales medias en cada sección referidas al $12 \%$ de humedad determinada mediante sonda. (Vigueta 1-9).

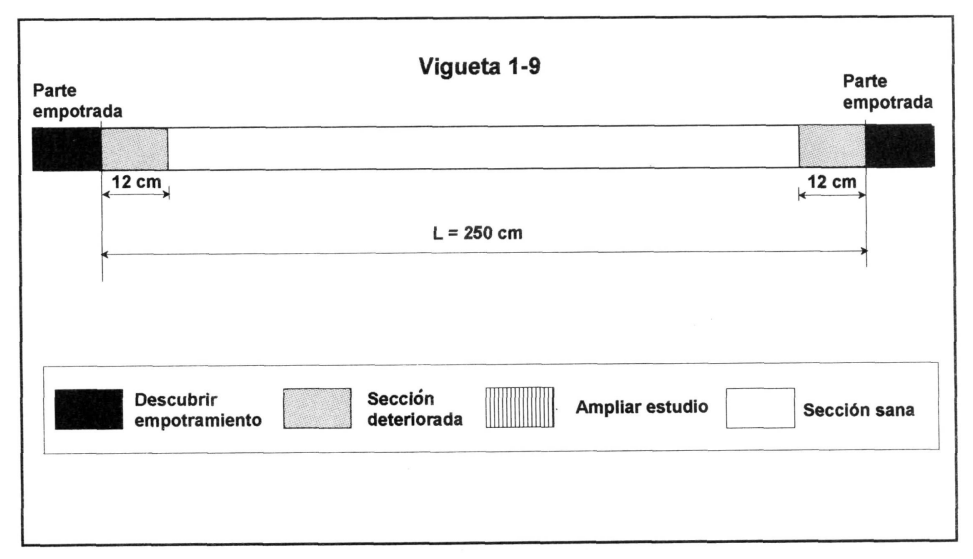

Fig. 11.- Estado de la viga 1-9.

$7^{\circ}$ ) Diagnóstico por vigas y habitaciones donde se indican (Fig. 11).

- Zonas sanas de las vigas.

- Zonas deterioradas.

- Zonas donde hay que ampliar el estudio. (c) Consejo Superior de Investigaciones Científicas Licencia Creative Commons 3.0 España (by-nc)
- Vigas en las que hay que descubrir las partes empotradas.

\subsection{Ensayos destructivos: Fase III}

Se han obtenido las tensiones de rōtura a flexión y los módulos de elasticidad estático a partir de los ensayos destructivos tanto para madera nueva como para madera vieja, cuyos resultados se dan en las tablas 4 y 5 . 
Tabla 4

Ensayo de flexión estática

\begin{tabular}{|c|c|c|c|c|c|}
\hline Probeta & $\begin{array}{l}\text { Carga de } \\
\text { rotura kp }\end{array}$ & Flecha $\mathrm{mm}$ & $\begin{array}{c}\sigma_{\mathrm{F}} \\
\mathrm{kp} / \mathrm{cm}^{2}\end{array}$ & $\begin{array}{c}E_{\mathrm{L}}^{\prime} \\
\mathrm{kp} / \mathrm{cm}^{2}\end{array}$ & Notas \\
\hline 1.a & 162 & 7,23 & 729 & 54.851 & $a=5^{\circ} *$ \\
\hline 1.b & 209 & 11,74 & 940 & 43.580 & $a=25^{\circ}$ \\
\hline $1 . c$ & 171 & 10,81 & 769 & 38.724 & $a=0^{\circ}$ \\
\hline 1.d & 232 & 10,96 & 1.044 & 51.819 & $a=0^{\circ}$ \\
\hline 1.e & 226 & 11,2 & 1.017 & 49.003 & $a=0^{\circ}$ \\
\hline 3.a & 214 & 10,98 & 963 & 47.711 & $a=5^{\circ}$ \\
\hline 3.b & 193 & 10,23 & 868 & 46.184 & $a=0^{\circ}$ \\
\hline 3.c & 157 & 10,44 & 708 & 36.814 & $a=0^{\circ}$ \\
\hline 3.d & 163 & 12,03 & 733 & 33.169 & $a=0^{\circ}$ \\
\hline 4.a & 234 & 11,29 & 1.053 & 50.728 & $a=0^{\circ}$ \\
\hline 4.b & 211 & 11,4 & 949 & 45.309 & $a=20^{\circ}$ \\
\hline 4.c & 197 & 11,5 & 886 & 41.753 & $a=45^{\circ}$ \\
\hline 4.d & 235 & 11,86 & 1.057 & 48.506 & $a=20^{\circ}$ \\
\hline 4.e & 247 & 12,0 & 1.111 & 50.220 & $a=0^{\circ}$ \\
\hline 5.a & 181 & 10,24 & 814 & 43.143 & $a=0^{\circ}$ \\
\hline 5.b & 107 & 6,67 & 481 & 39.269 & $a=0^{\circ}\left({ }^{*}\right)$ \\
\hline 5.c & 132 & 7,98 & 594 & 40.493 & $a=0^{\circ}\left({ }^{*}\right)$ \\
\hline 5.d & 181 & 10,26 & 814 & 43.186 & $a=20^{\circ}\left({ }^{*}\right)$ \\
\hline $5 . e$ & 172 & 9,75 & 774 & 43.185 & $a=20^{\circ}$ \\
\hline $6 . a$ & 186 & 13,10 & 837 & 34.656 & $a=0^{\circ}$ \\
\hline 6.b & 244 & 11,09 & 1.098 & 53.702 & $a=0^{\circ}$ \\
\hline 6.c & 216 & 11,50 & 972 & 45.978 & $a=20^{\circ}$ \\
\hline 6.d & 252 & 10,73 & 1.134 & 57.492 & $a=0^{\circ}$ \\
\hline $6 . e$ & 216 & 10,97 & 972 & 48.201 & $a=0^{\circ}$ \\
\hline 7.a & 154 & 8,45 & 693 & 42.121 & $a=0^{\circ}$ \\
\hline 7.b & 168 & 8,71 & 756 & 47.217 & $a=25^{\circ}$ \\
\hline 7.c & 138 & 7,75 & 621 & 43.589 & $a=0^{\circ}$ \\
\hline 7.d & 165 & 8,99 & 742 & 44.928 & $a=45^{\circ}$ \\
\hline $7 . e$ & 159 & 9,26 & 715 & 42.033 & $a=15^{\circ}$ \\
\hline $8 . a$ & 179 & 9,76 & 805 & 44.896 & $a=30^{\circ}$ \\
\hline 8.b & 163 & 10,76 & 733 & 37.084 & $a=0^{\circ}$ \\
\hline 8.c & 175 & 10,01 & 787 & 42.796 & $a=0^{\circ}$ \\
\hline 8.d & 164 & 9,76 & 738 & 41.134 & $a=40^{\circ}$ \\
\hline
\end{tabular}

Tabla 5

Ensayo de flexión estática

\begin{tabular}{||c|c|c|c|c|c|}
\hline Probeta $n^{\circ}$ & $\begin{array}{c}\text { Carga de } \\
\text { rotura kp }\end{array}$ & Flecha mm & $\begin{array}{c}\sigma_{\mathrm{F}} \\
\mathrm{kp} / \mathrm{cm}^{2}\end{array}$ & $\begin{array}{c}\mathrm{E}_{\mathrm{L}}^{\prime} \\
\mathrm{kp} / \mathrm{cm}^{2}\end{array}$ \\
\hline MVCA1 & 149 & 6,12 & 661,5 & 58.799 & $0^{\circ}$ \\
MVCA2 & 185 & 4,74 & 832,5 & 95.554 & $a=0^{\circ}$ \\
MVCA3 & 172 & 4,23 & 774 & 69.537 & $0^{\circ}$ \\
MVCA4 & 129 & 4,91 & 580,5 & 715 & $0^{\circ}\left(^{*}\right)$ \\
MVCA5 & 158 & 5,24 & 711 & 78.13 & $0^{\circ}$ \\
MVCA6 & 151 & 4,73 & 679,5 & $a=0^{\circ}$ \\
\hline
\end{tabular}

Notas:

$a=$ Ángulo de inclinación de las fibras de la probeta respecto al plano de flexión. * ${ }^{*}$ Rotura por un nudo.

$(*)=$ Probetas con nudos.

6. CORRELACIÓN DE MEDIDAS DE ULTRASONIDOS CON PARÁMETROS RESISTENTES. FASE IV

Sobre la base de datos creada con los resultados de ensayos destructivos y no destructivos se han obtenido las rectas de regresión que representan las relaciones que existen entre

(c) Consejo Superior de Investigaciones Científicas Licencia Creative Commons 3.0 España (by-nc) variables de ambos bloques de datos.

Madera nueva

1. Relación entre tensión de rotura a flexión $\left(\mathrm{kp} / \mathrm{cm}^{2}\right)$ y la velocidad de transmisión longitudinal $\mathrm{v}_{\mathrm{L}}(\mathrm{m} / \mathrm{sg})$ (Fig. 12). 


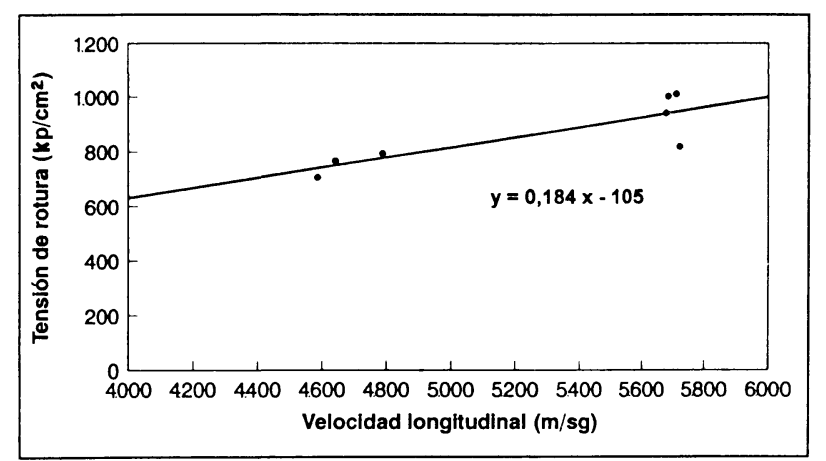

Fig. 12.- Relación entre latensión de rotura aflexión y velocidad longitudinal de transmisión de ultrasonidos.

\section{Datos estadísticos:}

\begin{tabular}{|c|c|c|c|c|c|c|c|c|c|c|}
\hline Serie & $\mathrm{N}^{\circ}$ val. & $\begin{array}{l}\text { Valor } \\
\text { más } \\
\text { bajo }\end{array}$ & $\begin{array}{l}\text { Valor } \\
\text { más } \\
\text { alto }\end{array}$ & $\begin{array}{l}\text { Valor } \\
\text { medio }\end{array}$ & Mediana & $\begin{array}{l}\text { Desv. } \\
\text { típica }\end{array}$ & $\begin{array}{l}\text { Coef. } \\
\text { corr. } \\
\text { R }\end{array}$ & $R^{2}$ & $\begin{array}{c}\text { Coef. } \\
\text { recta } \\
\text { A }\end{array}$ & $\begin{array}{c}\text { Coef. } \\
\text { recta } \\
\text { B }\end{array}$ \\
\hline$\sigma / V_{\mathrm{L}}$ & 7 & 705 & 1.011 & 862 & 818 & 112,5 & 0,836 & 0,699 & $-105,4$ & 0,18 \\
\hline
\end{tabular}

2. Relación entre módulos estáticos y dinámicos y la densidad. En este caso las correlaciones obtenidas son muy débiles.

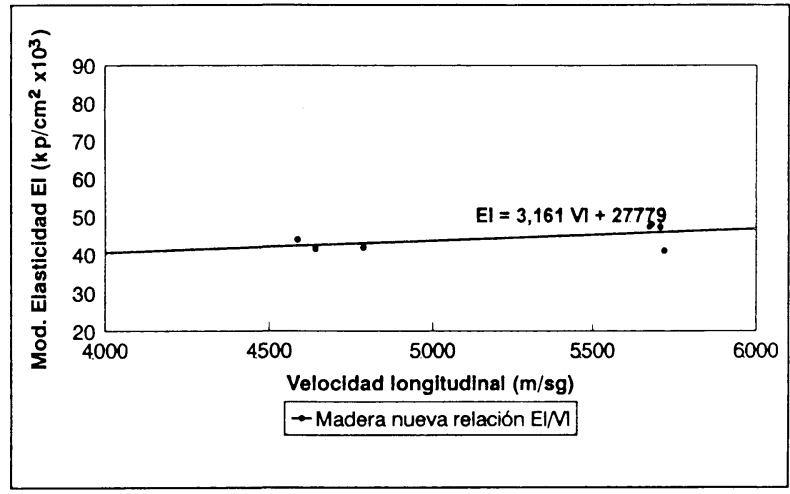

3. Relación entre el módulo de elasticidad estático y velocidad longitudinal de transmisión (Fig. 13).
Fig. 13.- Relación entre el módulo de elasticidad estático y la velocidad de transmisión longitudinal.

\section{Datos estadísticos:}

\begin{tabular}{||c|c|c|c|c|c|c|c|c|c||}
\hline Serie & $\begin{array}{c}\mathrm{N}^{\circ} \\
\text { val. }\end{array}$ & $\begin{array}{c}\text { Valor } \\
\text { más bajo }\end{array}$ & $\begin{array}{c}\text { Valor } \\
\text { más alto }\end{array}$ & $\begin{array}{c}\text { Valor } \\
\text { medio }\end{array}$ & $\begin{array}{c}\text { Desv. } \\
\text { típica }\end{array}$ & $\begin{array}{c}\text { Coef. } \\
\text { corr. } \\
\mathrm{R}\end{array}$ & $\mathrm{R}^{2}$ & $\begin{array}{c}\text { Coef. } \\
\text { recta A }\end{array}$ & $\begin{array}{c}\text { Coef. } \\
\text { recta } B\end{array}$ \\
\hline $\mathrm{E}_{\mathrm{L}} / \mathrm{V}_{\mathrm{L}}$ & 7 & 40.969 & 48.005 & 44.422 & $2.858,8$ & 0,566 & 0,3 & $2.797,1$ & 3,161 \\
\hline
\end{tabular}

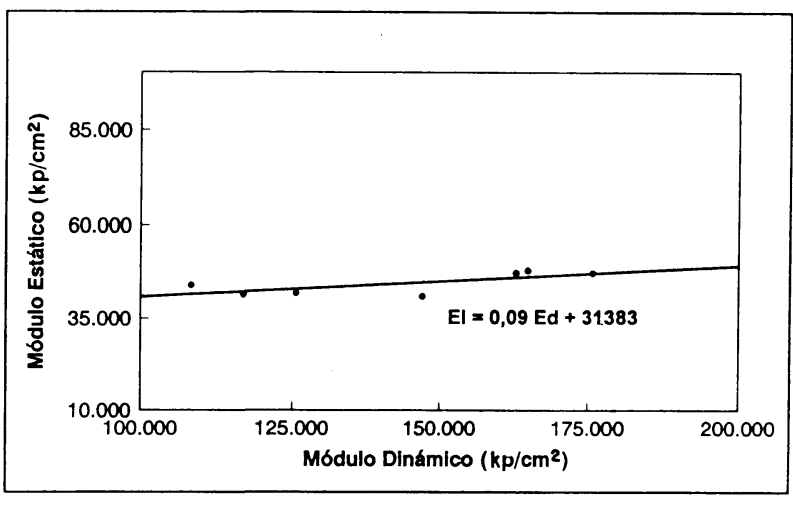

(c) Consejo Superior de Investigaciones Científicas Licencia Creative Commons 3.0 España (by-nc)
4. Relación entre el módulo de elasticidad estático y dinámico (Fig. 14).

Fig. 14.- Relación entre el módulo de elasticidäd estático y el módulo de elasticidad dinámico. 


\section{Datos estadísticos:}

\begin{tabular}{|c|c|c|c|c|c|c|c|c|c|c|}
\hline Serie & $N^{\circ}$ val. & $\begin{array}{c}\text { Valor } \\
\text { más } \\
\text { bajo }\end{array}$ & $\begin{array}{c}\text { Valor } \\
\text { más } \\
\text { alto }\end{array}$ & $\begin{array}{c}\text { Valor } \\
\text { medio }\end{array}$ & Mediana & $\begin{array}{c}\text { Desv. } \\
\text { tipica }\end{array}$ & $\begin{array}{c}\text { Coef. } \\
\text { corr. } \\
R\end{array}$ & $R^{2}$ & $\begin{array}{c}\text { Coef. } \\
\text { recta } \\
\text { A }\end{array}$ & $\begin{array}{c}\text { Coef. } \\
\text { recta } \\
B\end{array}$ \\
\hline$E_{\mathrm{L}} / E_{\mathrm{D}}$ & 7 & 40.909 & 48.005 & 44.222 & 43.977 & 2.858 & 0,566 & 0,3 & 2797,1 & 3,161 \\
\hline
\end{tabular}

\section{Madera vieja}

1. Relación entre tensión de rotura a flexión $\left(\mathrm{kp} / \mathrm{cm}^{2}\right)$ y la velocidad de transmisión longitudinal, $\mathrm{V}_{\mathrm{L}}(\mathrm{m} / \mathrm{sg})$ (Fig. 15).

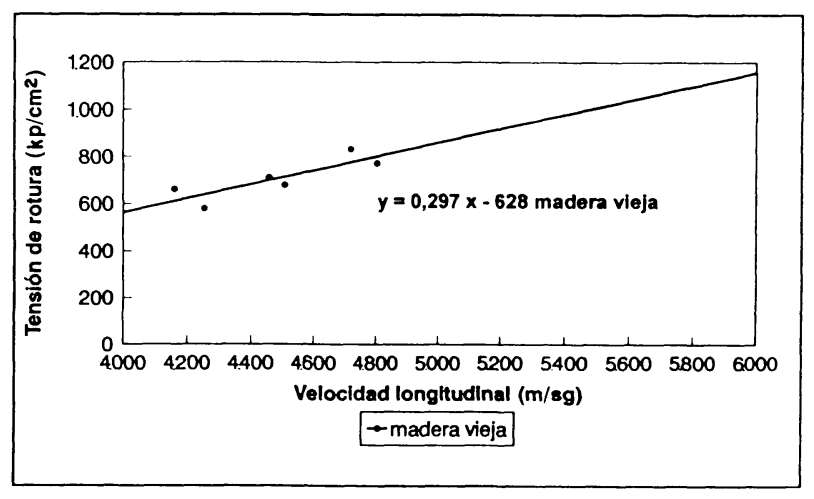

Fig. 15.- Relación entre la tensión de rotura a flexión y la velocidad longitudinal de transmisión del ensayo de ultrasonidos en madera vieja.

\section{Datos estadísticos:}

\begin{tabular}{||c|c|c|c|c|c|c|c|c|c|}
\hline $\begin{array}{c}\text { Serie } \\
\sigma / \mathrm{V}_{\mathrm{L}}\end{array}$ & $\begin{array}{c}\mathrm{N}^{\circ} \\
\text { val. }\end{array}$ & $\begin{array}{c}\text { Valor más } \\
\text { bajo }\end{array}$ & $\begin{array}{c}\text { Valor más } \\
\text { alto }\end{array}$ & $\begin{array}{c}\text { Valor } \\
\text { medio }\end{array}$ & $\begin{array}{c}\text { Desv. } \\
\text { típica }\end{array}$ & $\begin{array}{c}\text { Coef. } \\
\text { corr. } \\
\mathrm{R}\end{array}$ & $\mathrm{R}^{2}$ & $\begin{array}{c}\text { Coef. recta } \\
\mathrm{A}\end{array}$ & $\begin{array}{c}\text { Coef. } \\
\text { recta } \\
\mathrm{B}\end{array}$ \\
\hline MCVA & 6 & 580,5 & 832,5 & 695,1 & 80,7 & 0,84 & 0,70 & -628 & 0,29 \\
\hline
\end{tabular}

2. Relación entre el módulo de elasticidad estático y velocidad longitudinal de transmisión (Fig. 16).

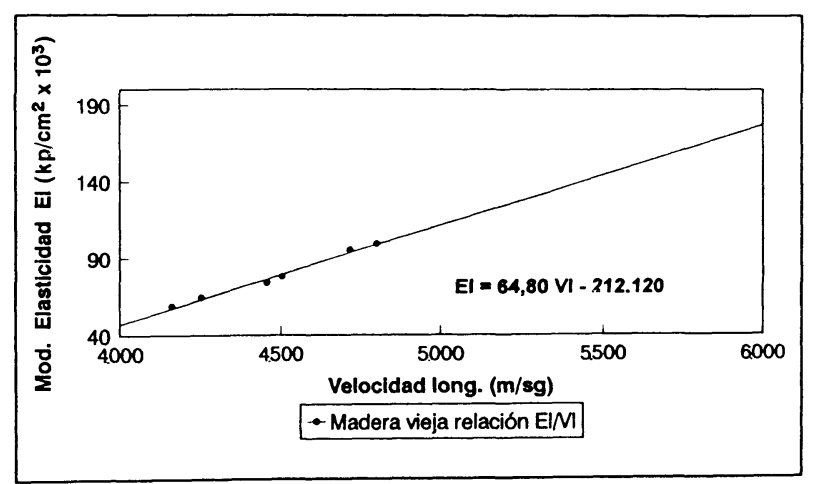

Fig. 16.- Relación entre el módulo de elasticidad estático y velocidad longitudinal de transmisión para madera vieja.

\section{Datos estadísticos:}

\begin{tabular}{||c|c|c|c|c|c|c|c|c|c|}
\hline Serie & $\begin{array}{c}N^{\circ} \\
\text { val. }\end{array}$ & $\begin{array}{c}\text { Valor más } \\
\text { bajo }\end{array}$ & $\begin{array}{c}\text { Valor más } \\
\text { alto }\end{array}$ & $\begin{array}{c}\text { Valor } \\
\text { medio }\end{array}$ & $\begin{array}{c}\text { Desv. } \\
\text { típica }\end{array}$ & $\begin{array}{c}\text { Coef. } \\
\text { corr. } \\
R\end{array}$ & $R^{2}$ & $\begin{array}{c}\text { Coef. recta } \\
A\end{array}$ & $\begin{array}{c}\text { Coef. } \\
\text { recta } \\
\text { B }\end{array}$ \\
\hline$E_{\mathrm{L}} / \mathrm{E}_{\mathrm{D}}$ & 6 & 58.799 & 99.537 & 78.361 & 14.970 & 0,99 & 0,98 & -212.120 & 64,80 \\
\hline
\end{tabular}




\section{ESTIMACIÓN DE LA RESISTENCIA A FLEXIÓN DE LA MADERA A PARTIR DE LAS MEDIDAS DE ULTRASONIDOS. FASE V}

Utilizando la recta de regresión que relaciona las velocidades longitudinales de ultrasonidos en madera nueva con la tensión de rotura del ensayo de flexión, hemos estimado la resistencia a flexión de la madera vieja a partir de los valores $\mathrm{V}_{\mathrm{L}}$ de ultrasonidos. Para comprobar la bondad del método se compara con la resistencia a flexión real obtenida del ensayo de rotura a flexión.

La estimación se hace sobre la hipótesis de suponer que se trata el conjunto de valores (muestra) obtenidos de una población con distribución normal y como el $n^{\circ}$ de valores de la muestra es pequeño $\mathrm{N} \geq 6$ y $\mathrm{N} \leq 30$, la estimación de los valores característicos (aquellos que nos dan un grado de confianza del $95 \%$ ) se hace utilizando la distribución $\mathrm{T}$ de Student, según la cual (Tabla 6):

valor característico $=$ valor medio -2 desviación tipica

Para el caso real de una obra de rehabilitación, al estar las cabezas de las vigas empotradas, no se poseen medidas de $\mathrm{V}_{\mathrm{L}}$, por loque utilizamos las velocidades cruzadas máximas $y$, mediante la relación obtenida en laboratorio, determinamos la velocidad longitudinal como sigue:

$$
\mathrm{V}_{\mathrm{L}}=\mathrm{V}_{\text {Cru Max. }} \times 1,19
$$

Los resultados conseguidos al aplicar esta metodología a la obra de rehabilitación son excelentes, ya que la tensión real obtenida del ensayo de rotura realizado sobre muestras de madera de la obra y la tensión básica estimada por el método propuesto tienen el mismo valor (la comparación se hace siempre para valores característicos) (Tabla 7).

De esta tabla, y para el valor característico de la tensión de rotura, podemos obtener la tensión básica estimada:

$$
\begin{aligned}
& \text { Tensión básica estimada }=\frac{\text { Valor característico }}{3,43}= \\
& =\frac{528,5}{3,43}=154,0 \mathrm{kp} / \mathrm{cm}^{2} \quad(\text { Ver Tabla } 7)
\end{aligned}
$$$$
\text { Tensión básica real }=\frac{\text { Valor característico }}{3,43}=
$$$$
=\frac{529}{3.43}=154.2 \mathrm{kp} / \mathrm{cm}^{2} \quad[\operatorname{Ver}(1) \text { Tabla 6] }
$$

Tabla 6

Relación entre valores de tensión de rotura para madera vieja obtenidos mediante la correlación con medida de ultrasonidos y los valores reales del ensayo de flexión

\begin{tabular}{||c|c|c|c||}
\hline $\begin{array}{c}N^{0} \text { de } \\
\text { probeta }\end{array}$ & $\begin{array}{c}\mathrm{V}_{\mathrm{L}} \mathrm{m} / \mathrm{sg} \\
\text { ultrasonidos }\end{array}$ & $\begin{array}{c}\text { Ensayo de flexión } \sigma \\
\mathrm{kp} / \mathrm{cm}^{2}\end{array}$ & $\begin{array}{c}\sigma \mathrm{kp} / \mathrm{cm}^{2} \\
\text { regresión }\end{array}$ \\
\hline MVCA1 & 4.161 & 661,5 & 660 \\
MVCA2 & 4.719 & 832,5 & 762 \\
MVCA3 & 4.803 & 774 & 778 \\
MVCA4( $\left.{ }^{*}\right)$ & 4.252 & 580,5 & 676 \\
MVCA5 & 4.456 & 711 & 714 \\
MVCA6 & 4.503 & 679,5 & 723 \\
\hline Valor medio & 4.482 & 706,5 & 719,2 \\
Desv. típica & 251,5 & 88,41 & 112,5 \\
Valor caract. & 3.979 & 529,68 & 449,2 \\
\hline T. básica $\sigma_{b}$ & & 154,2 & 144,0 \\
kp/cm & & real(1) & estimada (2) \\
\hline
\end{tabular}

(1 Tensión básica real (obtenida a partir de rotura a flexión de probetas) $=$ Valor característico / F

$F=$ coeficiente de seguridad $=3,43$

(2) Tensión básica estimada ( obtenida a partir de medida de ultrasonidos y correlación con tensión de rotura ) =

Valor característico / 3,43

(*) Probeta con un nudo en zona comprimida. 
Tabla 7

Tensiones de rotura estimadas a partir de valores de ultrasonidos: obra de rehabilitación de la c/ Narciso Campillo de Sevilla

\begin{tabular}{|c|l|l|l||}
\hline$N^{0}$ Viga & $\begin{array}{l}V_{\text {cruz max }} \\
(\mathrm{m} / \mathrm{sg})\end{array}$ & $\begin{array}{c}V_{L} \text { estimada } \\
(\mathrm{m} / \mathrm{sg})\end{array}$ & $\begin{array}{c}\text { Tensión de rotura } \\
\text { estimada } \mathrm{kp} / \mathrm{cm}^{2}\end{array}$ \\
\hline $2-2$ & 3.229 & 3.843 & 601,7 \\
$2-4$ & 3.879 & 4.616 & 743,9 \\
$2-9$ & 3.218 & 3.829 & 599,13 \\
$1-9$ & 3.721 & 4.427 & 709,1 \\
$1-10$ & 3.648 & $4.383,9$ & 701,2 \\
galería & 3.307 & $5.243(\mathrm{v}$ medio) & 859 \\
garaje & 3.844 & 4.574 & 736,2 \\
\hline Valor medio & $3.549,4$ & $4.416,5$ & 707,17 \\
Desv. típica $\sigma_{\mathrm{n}-1}$ & 289,35 & 486,9 & 89,51 \\
Val. característico & $2.968,3$ & $3.442,7$ & $\underline{528,15}$ \\
\hline
\end{tabular}

Estimación del módulo de elasticidad estático

En el caso del módulo de elasticidad estático lo que nos interesa conocer es el módulo de elasticidad medio. En este caso, la mejor estimación se tiene utilizando la recta de regresión que relaciona los valores de medidas de ultrasonidos con los módulos estáticos del ensayo de rotura para madera vieja. Se ha observado que, para la madera estudiada, la madera vieja tiene unos módulos de elasticidad más altos, es decir, es mucho más rígida que la madera nueva, quizás por efecto de un proceso de carga y descarga.
Supuestoéste que habrá que comprobar en fases posteriores de la investigación (Tabla 8).

$E_{L}^{\prime}($ valor medio estimado $)=74.064 \mathrm{kp} / \mathrm{cm}^{2}$

$E_{L}^{\prime}($ valor medio ensayo de flexión $)=78.361 \mathrm{kp} / \mathrm{cm}^{2}$

Si comparamos los valores resultantes de dicha tabla vemos como la estimación para $\mathrm{E}_{\mathrm{L}}{ }^{\prime}$, utilizando las medidas de ultrasonidos, es sólo inferior en un $5 \%$ al valor medio de los ensayos destructivos realizados para el mismo tipo de madera. Esta aproximación nos parece bastante aceptable.

Tabla 8

Módulo de Elasticidad estático estimado $E_{L}^{\prime}$ a partir de medidas de ultrasonidos: obra de Rehabilitación de la c/ Narciso Campillo de Sevilla

\begin{tabular}{|c|c|c|c|c|}
\hline$N^{0}$ Viga & $\begin{array}{l}V_{\text {cruz mox. }} \\
(\mathrm{m} / \mathrm{sg})\end{array}$ & $\begin{array}{c}V_{L} \text { estimada } \\
(\mathrm{m} / \mathrm{sg})\end{array}$ & $\begin{array}{c}E_{L}^{\prime} \text { estimado } \\
\left(\mathrm{kp} / \mathrm{cm}^{2}\right)\end{array}$ & $\begin{array}{l}E_{\mathrm{L}}^{\prime} \text { flexión } \\
\left(\mathrm{kp} / \mathrm{cm}^{2}\right)\end{array}$ \\
\hline $2-2$ & 3.229 & 3.843 & 36.906 & mvca \\
\hline $2-4$ & 3.879 & 4.616 & 86.996 & 58.799 \\
\hline $2-9$ & 3.218 & 3.829 & 35.999 & 95.554 \\
\hline $1-9$ & 3.721 & 4.427 & 74.749 & 99.537 \\
\hline $1-10$ & 3.648 & 4.383 & 71.898 & 64.315 \\
\hline galería & 3.307 & 5.243 (v. med) & 127.626 & 73.813 \\
\hline garaje & 3.844 & 4.574 & 84.275 & 78.148 \\
\hline Valor medio & $3.549,4$ & $4.416,5$ & 74.064 & -78.361 \\
\hline
\end{tabular}




\section{Estimación de la tensión admisible}

Además de obtener la tensión básica, hemos determinado cómo podemos obtener la tensión admisible utilizando las medidas de ultrasonidos, sin tener que realizar ninguna clasificación visual de defectos de una muestra representativa.

En el caso de madera nueva, la tensión admisible se define como aquellas tensiones que pueden resistir permanentemente y con seguridad, teniendo en cuenta los defectos e imperfecciones que contenga, es decir, su grado de calidad.

La tensión admisible se consigue a partir de la tensión básica de la madera, multiplicándola por un coeficiente corrector asociado a la clase o calidad de la madera.

De las medidas de velocidad de transmisión de ultrasonidos, realizadas sobre muestras de madera nueva, se puede deducir que cualquier defecto que tenga la madera, ya sea nudo o fenda, supone una reducción de la velocidad de propagación, siendo esta reducción proporcional al número de defectos.

Teniendo en cuenta los resultados de los ensayos realizados, consideramos que, si la velocidad longitudinal máxima cruzada obtenida representa una medida de la madera sin defectos, la velocidad longitudinal cruzada media representa una medida indirecta de los defectos que posee dicha madera. Por lo tanto, utilizando el mismo proceso empleado para obtener la tensión básica, pero partiendo de las medidas de las velocidades cruzadas medias en las vigas, obtendremos una estimación de la tensión admisible de la madera estudiada. El proceso utilizado se expone en la tabla 9 .

$$
\begin{aligned}
& \text { Tensión admisible }=\frac{\text { Valor característico }}{\text { Coeficiente de seguridad }} \\
& \text { Tensión admisible }=\frac{383,43}{3,43}=111,78 \mathrm{kp} / \mathrm{cm}^{2}
\end{aligned}
$$

Como el valor de la tensión básica de esta madera es:

$$
\text { Tensión básica }=154,2 \mathrm{kp} / \mathrm{cm}^{2}
$$

La reducción estimada debida a los defectos de la madera es de un $28 \%$, lo que expresado en términos de clase de madera sería una madera:

Clase II 70, según la norma UNE 5652572.

Otro camino más laborioso, y no por eso de mejor estimación, sería tomar las medidas de los defectos de la pieza al mismo tiempo que se realizan las mediciones de ultrasonidos.

Tabla 9

\begin{tabular}{|c|c|c|c|}
\hline$N^{0}$ Viga & $\begin{array}{l}V_{\text {cruz med. }} \\
(\mathrm{m} / \mathrm{sg})\end{array}$ & $\begin{array}{l}\mathrm{V}_{\mathrm{L}} \text { estimada } \\
(\mathrm{m} / \mathrm{sg})\end{array}$ & $\begin{array}{l}\text { Tensión adm. } \\
\text { estimada } \mathrm{kp} / \mathrm{cm}^{2}\end{array}$ \\
\hline $\begin{array}{c}2-2 \\
2-4 \\
2-9 \\
1-9 \\
1-10 \\
\text { garaje }\end{array}$ & $\begin{array}{l}2.614 \\
3.132 \\
2.842 \\
3.055 \\
3.224 \\
3.844\end{array}$ & $\begin{array}{l}3.110 \\
3.727 \\
3.381,9 \\
3.635 \\
3.836 \\
4.574\end{array}$ & $\begin{array}{l}454,4 \\
565,4 \\
503,18 \\
548,9 \\
585,08 \\
717,98\end{array}$ \\
\hline $\begin{array}{c}\text { Valor medio } \\
\text { Desv. típica } \sigma_{n-1} \\
\text { Val. característico }\end{array}$ & $\begin{array}{c}3.188,5 \\
417,77 \\
2.352,9\end{array}$ & $\begin{array}{c}3.710,65 \\
497,17 \\
2.716,3\end{array}$ & $\begin{array}{r}562,49 \\
89,53 \\
383,43 \\
-\end{array}$ \\
\hline
\end{tabular}

Tensiones admisibles estimadas a partir de ultrasonidos: obra de Rehabilitación de la c/ Narciso Campillo de Sevilla 


\section{CONCLUSIONES}

\section{Ensayos en madera nueva en laboratorio}

Relación entre las velocidades de transmisión según las distintas direcciones

- Los resultados de los ensayos nos indican que la relación que existe entre la velocidades de transmisión para distintas posiciones de la fibra de la madera es una relación que se mantiene de manera casi constante, independiente de las dimensiones transversales de las probetas.

- Se puede considerar que la relación entre velocidades de transmisión es una constante, independientemente de la humedad a la que se encuentre la muestra.

- Hemos visto que para el tipo de madera estudiada (probetas de madera nueva de Pino de Flandes) la velocidad de transmisión en la dirección radial $\left(\mathrm{V}_{\mathrm{N}}\right)$ es un $40 \%$ mayor que en la dirección tangencial.También podemos estimar que el valor medio de la relación entre $\mathrm{V}_{\mathrm{L}} / \mathrm{V}_{\mathrm{N}}$ es de $2,46 \mathrm{y}$ que el valor medio de la relación entre $\mathrm{V}_{\mathrm{L}} / \mathrm{V}_{\mathrm{T}}$ es de 3,44 , en ambos casos con una desviación típica de 0,2 .

- El valor medio de la relación entre la velocidad longitudinal media en el sentido de la fibra y la velocidad máxima cruzada es de $\mathrm{V}_{\mathrm{L}} / \mathrm{V}_{\text {Cruzmáx. }}=1,59$, para una desviación típica de 0,2 . Esta relación nos va a permitir obtener de una manera indirecta la velocidad longitudinal cuando no es posible acceder a los extremos empotrados de una pieza (caso de vigas empotradas en obras de rehabilitación).

- Aprovechando las diferencias sustanciales entre las velocidades de transmisión, normales y tangenciales, hemos diseñado un método que, mediante la realización de cuatro medidas transversales cruzadas, nos permite afirmar que los anillos de crecimiento en esa sección son normales a la dirección en la cual se registra la máxima velocidad de transmisión.

Influencia de la humedad de la madera en las velocidades de transmisión

- El efecto del incremento de humedad en el intervalo del $12 \%$ al $28 \%$ (madera no saturada) tiene más influencia en la velocidad normal a la fibra que en la velocidad longitudinal.

- Del estudiode la relación entre la velocidad de transmisión (tanto normal como longitudinal) y la humedad para una misma probeta de madera se tiene que:

- La correlación es muy alta para $\mathrm{H}<28 \%$, es decir, hay mucha influencia de la humedad en las velocidades de transmisión, no así para humedades mayores del $28 \%$ (madera saturada), donde se obtienen rectas casi horizontales, con correlaciones muy débiles.
- Según el estudio realizado, proponemos como rectas de ajuste de velocidad, para una humedad cualquiera, las rectas siguientes:

- Para medidas de velocidad longitudinal $\mathrm{V}_{\mathrm{L}}(\mathrm{m} / \mathrm{s})$

$$
\begin{gathered}
\text { Si } H<28 \% \\
V_{L H i}=-39 H_{i}+V_{L H 0} \\
\text { Si H }>28 \% \\
V_{L H I}=-0,73\left(28-H_{i}\right)+V_{L H 28}
\end{gathered}
$$

- Para medidas de velocidad normal a la fibra $V_{N}(\mathrm{~m} / \mathrm{s})$

$$
\begin{aligned}
& \text { Si } H<28 \% \\
& V_{\mathrm{NHi}}=-27,5 \mathrm{H}_{\mathrm{i}}+\mathrm{V}_{\mathrm{NH} 0} \\
& \mathrm{Si} \mathrm{H}>28 \% \\
& \mathrm{~V}_{\mathrm{NHI}}=-0,46\left(28-\mathrm{H}_{\mathrm{i}}\right)+\mathrm{V}_{\mathrm{NH} 28}
\end{aligned}
$$

Siendo:

$\mathrm{H}_{\mathrm{I}}$ : Humedad en $\%$.

$\mathrm{V}_{\mathrm{LHI}}$ : Velocidad longitudinal de transmisión para una humedad $\mathrm{H}_{\mathrm{I}}$.

$\mathrm{V}_{\mathrm{LH} 0}$ : Velocidad longitudinal de transmisión para una humedad del $0 \%$

$\mathrm{V}_{\text {LH28 }}$ : Velocidad longitudinal de transmisión para una humedad del $28 \%$.

$\mathrm{V}_{\mathrm{NH}}$ : Velocidad normal de transmisión para una humedad $\mathrm{H}_{1}$

$\mathrm{V}_{\mathrm{NH} \mathrm{O}}$ : Velocidad normal de transmisión para una humedad del $0 \%$

$\mathrm{V}_{\mathrm{NH} 28}$ : Velocidad normal de transmisión para una humedad del $28 \%$.

\section{Ensayos de madera vieja en una obra de rehabilitación}

Teniendo en cuenta las conclusiones anteriores obtenidas en laboratorio, se ha desarrollado una metodología original, usando la técnica de los ultrasonidos, que nos permite detectar estados de deterioro y pudrición de la madera de vigas de forjados en una obra de rehabilitación.

La metodología propuesta aplicada a un caso concreto de rehabilitación nos permite establecer las siguientes conclusiones:

- La inspección visual no es suficiente para determinar la existencia de zonas deterioradas en un forjado de vigas de 
maderay, en muchos casos, puede llevar a conclusiones erróneas. Es necesario utilizar una técnica de inspección complementaria como la que proponemos.

- Una bajada de velocidad de transmisión en la dirección transversal a las vigas supone (siempre que estemos comparando valores referidos a la misma humedad) una pérdida de peso de la madera en esa zona y, por lo tanto, un estado de deterioro y como consecuencia una pérdida de resistencia.

- Se ha estimado para la pudrición cúbica, que es la que aparece en las cabezas de las vigas empotradas y en las zonas adyacentes, que una bajada del $10 \%$ es ya lo suficientemente importante como para actuar sobre la zona. Para estudiar esta bajada de velocidad se toma como valor de referencia de madera sana el valor máximo de la velocidad media de transmisión de ultrasonidos en el tercio central, pues es en esta zona donde las vigas se encuentran en mejor estado.

- Todas las mediciones de velocidades de transmisión deben estar referidas a una misma humedad (normalmente al $12 \%$ ). Para ello se utilizarán las ecuaciones aportadas en este trabajo. Es conveniente la utilización de dos aparatos de medida: la sonda nos da medidas superficiales de la humedad y el M49 medidas más globales.

- Para una determinada viga o vigueta, la dirección de la fibra viene dada uniendo los valores más altos de velocidades de transmisión en cada una de las secciones estudiadas. Esto nos permite detectar deterioros puntuales ya que, en dos secciones contiguas y en puntos situados en la misma posición en relación a la fibra, no debe haber cambios importantes en la medición de la velocidad.

- Las medidas de velocidades longitudinales, lo mismo que las normales, también sufren una disminución apreciable cuando en su trayectoria atraviesan zonas con pudrición. Esto se comprobó en vigas que estaban desmontadas, pero este sistema no es operativo puesto que no se puede realizar la medición cuando las vigas están empotradas.

- Cuando no es posible realizar medidas de transmisión en la dirección de la fibra (velocidad longitudinal) se pueden sustituir por medidas de velocidades longitudinales cruzadas, realizadas entre caras opuestas, como se especifica en el método de ensayo correspondiente.

- Es posible establecer, utilizando la técnica de ultrasonidos, un diagnóstico de las vigas de madera por zonas, distinguiendo la madera deteriorada o con un inicio de estado de pudrición. Lo que nos llevará a determinar qué zonas es necesario reparar o sustituir o en cuáles es necesario ampliar el estudio.
Obtención de parámetros resistentes de la madera vieja a partir de las medidas de ultrasonidos

- Es posible obtener con un grado de confianza suficiente los valores que caracterizan la resistencia de la madera vieja de una obra de rehabilitación sin tener que realizar ensayos destructivos.

Esto se consigue teniendo en cuenta las relaciones establecidas para el mismo tipo de madera (pino flandes) en el caso de madera nueva, entre las medidas de ultrasonidos longitudinales y la tensión de rotura a flexión.

\section{Tensión básica}

Los valores de la tensión básica, determinados a partir de las medidas de ultrasonidos y con las correlaciones aportadas, son valores característicos (nivel de confianza del $95 \%$ ) y para su obtención suponemos la distribución de probabilidad $\mathrm{T}$ de Student, ya que el número de valores que manejamos no es muy elevado. $(\mathrm{N}>6, \mathrm{~N}<30)$.

Para superar la dificultad de determinar la velocidad de transmisión según la dirección de la fibra se han determinado velocidades cruzadas en el tercio central de la viga.

La aplicación de esta metodología a la obra de rehabilitación estudiada nos da una aproximación excelente al valor de tensión básica obtenido por el método tradicional de rotura de probetas a flexión estática: menos del $0,8 \%$ de error por defecto

$$
\begin{aligned}
& \sigma_{\text {básca estimada }}=154 \mathrm{kp} / \mathrm{cm}^{2} \\
& \sigma_{\text {básca real }}=154,2 \mathrm{kp} / \mathrm{cm}^{2}
\end{aligned}
$$

\section{Tensión admisible}

Se propone como método para determinar la tensión admisible de la madera vieja de la obra de rehabilitación la utilización de la velocidad cruzada media como medida indirecta de los defectos que posee la madera y, a partir de ella, obtener la tensión admisible por el mismo método que se ha aplicado para la tensión básica

\section{Módulo de elasticidad estático}

Como método para obtener el módulo de elasticidad estático de la madera vieja se propone la utilización de la ecuación siguiente que relaciona dicho módulo con la velocidad longitudinal de transmisión:

$$
\mathrm{E}_{\mathrm{L}}=64,80 \mathrm{~V}_{\mathrm{L}}-212 . \overline{1} 20
$$


Se utilizarán las velocidades longitudinales deducidas de la velocidades cruzadas máximas.

En este caso lo que nos interesa es la determinación del valor medio del módulo, no un valor característico
El grado de aproximación alcanzado en el caso de la obra de rehabilitación estudiada es de un $5 \%$ por defecto respecto al valor del modulo real, determinado a partir de los ensayos, de flexión estática para las probetas de madera vieja.

\title{
BIBLIOGRAFIA
}

(1) LEE, I.D.G.: "Testing for Safety in Timber Structures Paper 12". Symposium of Non-destructive Testing of ('oncrete and Timber. Inst. of Civil Engineers London ( 1969) p. 115 -118.*

(2) AGGOUR, M.S., RAGAB A.M., y E. J. WHITE J.R.: "Determination of In-Place Timber Piling Strenght". Transportation Research Record n ${ }^{a} 962$. (1984) pp. 69-77.*

(3) ARITA, K., y KURATANI, K.: "Wooden Pole Tester for Determining Strength of Decayed Wooden Poles". (1984).*

(4) WAYNE, W., y WILCOX.: "Detection of early stages of wood decay with ultrasonic pulse velocity". Forest Products Journal XXXVIII n ${ }^{\circ} 5$ (1988) pp. 68-73. *
(5) SANDOZ, J.L. "Moisture Content and Temperature Effect on Ultrasonic Timber Grading" Wood Sci. Technol. $\mathrm{n}^{\circ}$ 27(5) (1993) pp. 373-380.

(6) ARRIAGA, F. et al.: "Evaluación de la capacidad portante de forjados antiguos con vigas de madera". Rev. AITIM.(1993) pp .51-59.*

(7) PALAIA, L. y GALVAÑ, V. et al: "Estimación de la restauración de la madera en edificios antiguos por medio de técnicas ultrasónicas" Primer C'ongreso Nacional de Tecnologia en la Arquitectura E.T.S.A. de Madrid. noviembre 1994. pp.63-77.

\section{publicación del IETCC/CSIC}

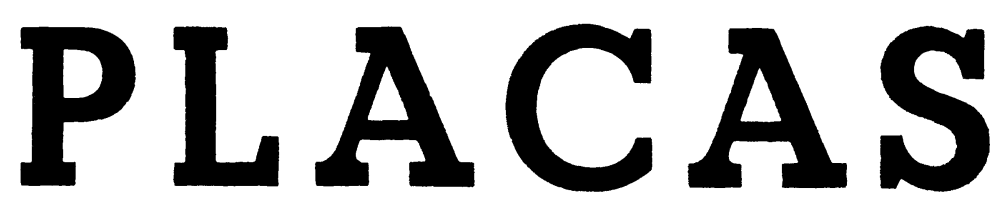

\author{
K. Striglat gy H. Wippel \\ Drs. Ingenieros \\ Traducción de Juan Batanero \\ Dr. Ingeniero de Caminos \\ con la colaboración de \\ Francisco Morán \\ Ingeniero de Caminos
}

\begin{abstract}
Este libro, cuidadosa y magníficamente editado, reúne, quizás, la más completa colección conocida de tablas para placas, por los numerosos casos de vinculación y de carga estudiados y por la abundancia de relaciones de dimensión y de datos ofrecidos, que cubren prácticamente todo el campo de las losas en edificación. Permite desarrollar, con comodidad, rapidez y una aproximación suficiente, los cálculos de dimensionamiento y comprobación, obviando las dificultades que como es sabido, presenta el desarrollo numérico de los métodos de cálculo de estos elementos, evitando enojosas operaciones.

Trata la obra sobre "Zonas de Placas", "Placas sobre apoyos puntuales", "Placas apoyadas en dos, tres y cuatro bordes" y "Placas apoyadas elásticamente", tipos que en la actualidad disponian de una documentación, incompleta o nula, para la determinación de esfuerzos. Los corrimientos de la placa, como valores previos para la determinación de los momentos, han sido obtenidos por medio del Cálculo de Diferencias, método que se ha comprobado como suficientemente satisfactorio, aún en su forma simple, aplicado con un cierto control.
\end{abstract}

Un volumen encuadernado en tela, de $30,5 \times 23,5 \mathrm{~cm}$, compuesto de 92 págs. Madrid, $\mathbf{1 9 6 8}$.

Precios: España, 2.500 ptas.; extranjero, $\$ 36.00$. 